\title{
EFFECT OF GLUTATHIONE REDOX SYSTEM ON LYSOZYME REFOLDING IN SIZE EXCLUSION CHROMATOGRAPHY
}

\author{
S. S.-S. WANG, C.-K. CHANG, M.-J. PENG and H.-S. LIU* \\ Department of Chemical Engineering, National Taiwan University, Taipei, Taiwan
}

\begin{abstract}
$\mathrm{T}$
he refolding step is of key importance for industrially produced protein aggregates to regain their biological activities. A number of strategies, including the direct dilution and chromatographic methods, have been utilized to improve the performance of the refolding process; however, refinement and advances on these methods are ongoing. Using lysozymes as a model system, we presented a study on the effects of redox system GSH/ GSSG on protein refolding process in size exclusion chromatography (SEC). Attempts were first made to understand how redox constituents of refolding buffer affected the direct dilution or SEC refolding performance, and then to compare the operating mechanisms between the direct dilution and SEC refolding methods. Our results showed that, the combination of reduced as well as oxidized forms of glutathione was able to provide a favourable environment for protein renaturation. The performance of denatured protein refolding could be optimized via an appropriate choice of the relative distribution between GSH and GSSG. Moreover, our research further indicated that SEC packing provided an inhibitory action against aggregation, especially at lower dilution factors (or higher protein concentrations). We believe that the outcome from this study may facilitate the development of a more effective strategy for protein refolding processes.
\end{abstract}

Keywords: lysozyme; refolding; redox system; size exclusion chromatography.

\section{INTRODUCTION}

With the advent of genetic engineering, the formation of many recombinant engineered proteins can be achieved via their over-expression in bacteria. Large quantities of these engineered proteins can then be produced in rapid and inexpensive industrial bioprocesses. However, during the production process, the expression of proteins of interest often results in the aggregation of inactive intracellular deposits (i.e., inclusion bodies) which need to be refolded to regain their activities (Buchner et al., 1992). The recovery of active proteins from inclusion bodies is composed of three steps: isolation and washing of deposits, solubilization of aggregated protein, and renaturation/refolding of the solubilized proteins (Clark, 2001). While the efficiency of the first two steps could be fairly high, the major challenge is to develop optimum processes whereby the formation of inactive misfolded aggregates can be avoided during the renaturation/refolding step.

In general, various denaturant species, for instance, urea and guanidine hydrochloride, were employed to solubilize

*Correspondence to: Professor H.-S. Liu, Department of Chemical Engineering, National Taiwan University, No.1, Sec.4, Roosevelt Road, Taipei, Taiwan 10617.

E-mail: hsliu@ntu.edu.tw aggregates. Techniques such as direct dilution (Chang et al., 2004; Goldberg et al., 1991; Lai and Middelberg, 2002; Wright and Serpersu, 2004), dialysis (West et al., 1998), immobilization of the denatured protein onto a solid support (Kim et al., 1997; Negro et al., 1997), size exclusion chromatography (Batas and Chaudhuri, 1996; Hamaker et al., 1996; Liu and Chang, 2003; Wang et al., 2005), and ion-exchange chromatography (Kweon et al., 2004; Li and $\mathrm{Su}, 2002 \mathrm{a}$, b; Li et al., 2002) have been reported as potential methods to renature/refold the solubilized protein through decreasing the denaturant concentration or removing the excess denaturants. Due to its simplicity and scale-invariant feature, the direct dilution of denatured proteins with proper refolding buffer has been the commonly used method in most refolding studies.

Evidence showed that the aggregation pathway is governed by intermolecular interactions between hydrophobic patches on various protein molecules, and therefore, is strongly associated with protein concentrations. This undesired aggregation phenomenon follows a higher order kinetic scheme that competes with the first order reaction of correct refolding. As a result, the key to reduce aggregation is to circumvent the intermolecular interaction by decreasing protein concentration. Extensive efforts have been directed toward preventing such unwanted side reaction in the refolding step. Several methods have been used to 
improve the yield of protein renaturation, such as addition of detergents (Goto et al., 2000a; Wetlaufer et al., 1995), cyclodextrins (Karuppiah and Sharma, 1995), liposome (Zardeneta and Horowitz, 1994), acetone, acetonamide, urea derivatives (Yasuda et al., 1998), and ammonium sulphate (Maeda et al., 1996) to prevent aggregations, use of polyethylene glycol to stabilize refolding intermediates (Cleland et al., 1992a, b), and passage though a column with immobilized molecular chaperones (Dong et al., 2000; Mannen et al., 2001). On the other hand, because the refolding and the separation simultaneously can be feasibly achieved within the packing matrices, size exclusion chromatography (SEC) has also been considered as an alternative buffer exchange method to enhance protein refolding (Batas and Chaudhuri, 1996; Lanckriet and Middelberg, 2004; Liu and Chang, 2003; Wang et al., 2006).

It is well recognized that disulphide bonds stabilize the correct conformation of proteins. Hence, the formation of proper disulfide bonds plays a pivotal role during the course of protein folding/refolding. The influence of redox agents in buffers (redox environment) on refolding yield has been addressed and examined on a number of proteins (Fischer et al., 1993; Fischer, 1994; Zapun and Creighton, 1994). The formation and reshuffling of disulphide bonds can be promoted via the addition of so-called oxido-shuffling reagents in refolding buffers (Clark et al., 1998; Hevehan and Clark, 1997). The widely used oxido-shuffling reagents are the reduced and oxidized forms of glutathione, termed GSH and GSSG, respectively (Clark, 2001; Frand et al., 2000) that the performance of refolding is highly correlated with the relative compositions between the oxidized and reduced counterparts (Bulaj, 2005; Hevehan and Clark, 1997).

Utilizing lysozymes as a model system, we describe here a study on the effects of redox system GSH/GSSG on protein refolding process in SEC. Attempts were made not only to understand how redox constituents of refolding buffer affect the direct dilution or SEC refolding performance, but also to elucidate the insight in operating mechanisms between the direct dilution and SEC refolding methods. Our results show that, the combination of reduced as well as oxidized forms of glutathione was able to provide a favourable refolding environment. The performance of denatured protein renaturation could be optimized via a proper choice of the relative distribution between GSH and GSSG. Additionally, our data further pointed out that SEC provided an inhibitory action against aggregation, especially at lower dilution factors (or higher protein concentrations). It is our belief that the outcome from this study may facilitate the development of a more effective strategy for protein refolding processes.

\section{MATERIALS AND METHODS}

\section{Materials}

Hen egg white lysozyme (HEWL; EC 3.2.1.17), hydrochloric acid $(\mathrm{HCl})$ and di-potassium hydrogen phosphate $\left(\mathrm{K}_{2} \mathrm{HPO}_{4}\right)$ were purchased from Merck (Germany). Dithiothreitol (DTT), Micrococcus lysodeikitus dried cells, oxidized glutathione (GSSG), reduced glutathione (GSH), and tris-(hydroxymethyl) aminomethane were purchased from Sigma (USA). Urea, EDTA, and potassium di-hydrogen phosphate $\left(\mathrm{KH}_{2} \mathrm{PO}_{4}\right)$ were obtained from Hayashi Pure Chemical Ind., Co., Ltd (Japan). All other chemicals were of analytical grade.

\section{Preparation of Denatured Lysozyme Sample Solution}

Different amounts of HEWL lysozyme were dissolved in $0.1 \mathrm{M}$ Tris- $\mathrm{HCl}$ solution to prepare lysozyme stock solutions. Denatured lysozyme sample solutions were made by mixing the lysozyme stock solution with the buffer solution at $\mathrm{pH}$ of 8.6 (0.1 M Tris-HCl, $1.25 \mathrm{mM}$ EDTA, $10 \mathrm{M}$ urea, and $0.0125 \mathrm{M}$ DTT). The activity of the denatured lysozyme sample solutions was observed to reach zero after storing at room temperature for $24 \mathrm{~h}$ and the mixture was then used for the following refolding experiments.

\section{Lysozyme Concentration and Activity Assays}

Lysozyme concentration was measured spectrophotometrically at the wavelength of $280 \mathrm{~nm}$. The activity of lysozyme sample solutions was determined by measuring the decrease in absorbance at $450 \mathrm{~nm}$. Under the $\mathrm{pH}$ of 6.2 and $25^{\circ} \mathrm{C}$, a $2.5 \mathrm{ml}$ volume of $M$. lysodeikticus suspension $\left(0.2 \mathrm{mg} \mathrm{ml}^{-1}\right)$ in $0.067 \mathrm{M}$ potassium phosphate was used as the substrate solution to react with $40 \mu \mathrm{l}$ of protein sample. One unit of activity corresponds to an absorbance decrease of 0.001 per min. The absorbance measurement was performed using the Spectronic Genesys 5 spectrophotometer (Spectronic Instrument, USA).

\section{Determination of Concentration of Insoluble Aggregates}

The denatured protein sample solution at $0.5 \mathrm{mg} \mathrm{ml}^{-1}$ was first centrifuged at $12000 \mathrm{rpm}$ for $15 \mathrm{~min}$ to remove the protein pellet/aggregates. The protein concentration of resulting supernatant (soluble lysozyme) was measured spectrophotometrically at the wavelength of $280 \mathrm{~nm}$. The protein content of aggregates was further determined by subtracting the concentration of soluble lysozyme from the denatured lysozyme concentration $\left(0.5 \mathrm{mg} \mathrm{ml}^{-1}\right)$.

\section{Refolding of Fully Reduced Lysozyme}

Two methods, direct dilution and size exclusion chromatography methods, were used for renaturation in this study. The performance of the refolding efficiency was assessed by the ratio of the total activity recovered after refolding to that of the native lysozyme before denaturation.

\section{Direct dilution method}

Following denaturation, the renaturation step was initiated by a rapid 10,43 or 600 -fold dilution of the denatured lysozyme sample solution in the refolding buffer of $\mathrm{pH} 8.2$ consisting of $0.1 \mathrm{M}$ Tris- $\mathrm{HCl}, 1 \mathrm{mM}$ EDTA, 0 or $2 \mathrm{M}$ urea, and various concentrations of GSH and GSSG.

\section{SEC method with redox gradient}

The high performance liquid chromatography (Waters) was equipped with a dual $\lambda$ absorbance detector (Waters 2487) and a binary HPLC pump (Waters 1525). To 
generate various redox environments, different combinations of two mobile phase solutions, namely buffer A and buffer $\mathrm{B}$, were used for size exclusion chromatography refolding with linear glutathione gradient. Apart from the general components, $2 \mathrm{M}$ urea, $0.2 \mathrm{M} \mathrm{NaCl}$, $1 \mathrm{mM}$ EDTA and $0.1 \mathrm{M}, \mathrm{pH} 8.2 \mathrm{Tris}-\mathrm{HCl}$, three different combinations of redox-associated constituents are: (1) $3.6 \mathrm{mM}$ GSH and $1.8 \mathrm{mM} \mathrm{GSSG}$; (2) $1.2 \mathrm{mM} \mathrm{GSH} /$ $1.2 \mathrm{mM}$ GSSG and $3 \mathrm{mM}$ GSH/0.3 mM GSSG; (3) $1.2 \mathrm{mM} \mathrm{GSH} / 1.2 \mathrm{mM}$ GSSG and No GSH and/or GSSG. In SEC operation, a Superdex 75(10/30) prepacked column was first equilibrated with buffer A. After equilibration, the sample solution was eluted through the column by gradually increasing the ratio of buffer A/buffer B from $0: 100$ to $100: 0$ at $0.5 \mathrm{ml}$ $\min ^{-1}$ within $1,5,10$ or 20 min of gradient time. The refolding environment was dynamically adjusted via the combination of mobile phase buffers and gradient duration. After introducing a linear glutathione gradient, a $12.5 \mu \mathrm{l}$ or $200 \mu \mathrm{l}$ aliquot of denatured lysozyme at $10 \mathrm{mg} \mathrm{ml}^{-1}$ or $20 \mathrm{mg} \mathrm{ml}^{-1}$ was loaded at the end of the gradient, where the mobile phase was buffer B. Owing to its large molecular mass of 14300 , the denatured proteins moved faster than the gradient formed by small molecules of glutathione (GSH or GSSG) inside of the column. Therefore the denatured protein could pass along the gradient and promote the correct disulphide bond formation.

\section{RESULTS}

\section{Effect of Constituents on Refolding of Denatured Lysozyme via Direct Dilution Method}

We first examined the effect of composition of refolding buffer on the performance of lysozyme renaturation in the direct dilution method. The overall refolding performance was assessed by the amounts (or concentrations) of insoluble aggregates and active lysozyme formed after refolding. Aggregates were determined by subtracting soluble protein, by OD280 after centrifugation, from initial protein amount. Our data indicated that, when the denatured lysozyme sample solution was diluted from 5 to $0.5 \mathrm{mg} \mathrm{ml}^{-1}$, the amount of aggregated species decreased as the concentration of urea rose from 0 to $2 \mathrm{M}$ after one day of renaturation in the Tris- $\mathrm{HCl}$ buffer solution (see Table 1). However, no lysozyme activity was recovered after the renaturation process regardless of the concentration of urea in use (see Table 1). We next investigated if the refolding performance was affected by the presence of redox reagents (GSH/GSSG) in the refolding buffer. As demonstrated in Table 1, the refolding performance was found to be strongly dependent on the relative concentrations of GSH and GSSG. Compared with 1.2 mM GSH alone, a better refolding performance (less aggregates and more active proteins formed) was observed at $1.2 \mathrm{mM}$ GSSG in Tris- $\mathrm{HCl}$ buffer without urea. Moreover, the refolding buffer containing redox counterparts initially at $1.2 \mathrm{mM}$ $\mathrm{GSH} / 1.2 \mathrm{mM}$ GSSG exhibited a maximal active protein concentration $\left(0.24 \mathrm{mg} \mathrm{ml}^{-1}\right)$ as well as minimal aggregate concentration $\left(0.1 \mathrm{mg} \mathrm{ml}^{-1}\right)$ among all cases in the study.

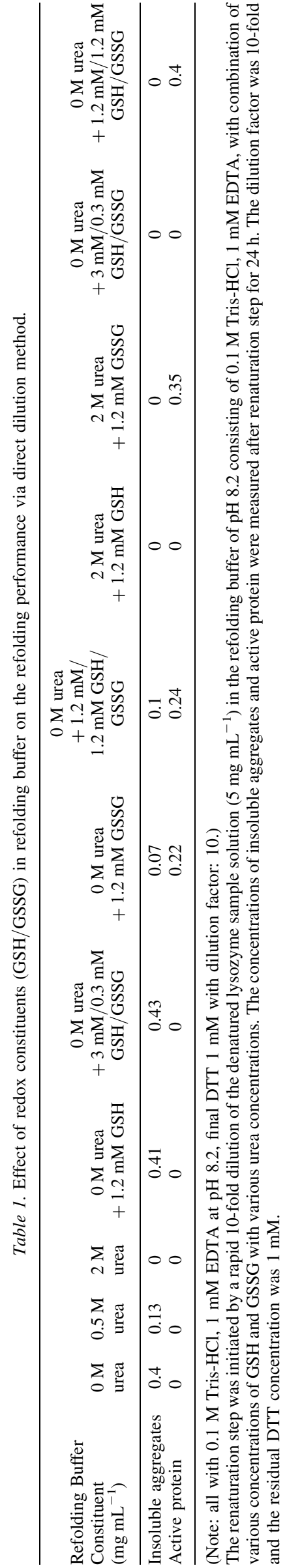


As a complement of the aforementioned set of experiments, we also performed the direct dilution refolding of denatured lysozyme using Tris- $\mathrm{HCl}$ in the presence of $2 \mathrm{M}$ urea (Table 1). Results showed that the insoluble aggregates disappeared in all cases upon addition of $2 \mathrm{M}$ urea in the refolding buffer (pictures not shown), suggesting the role of $2 \mathrm{M}$ urea in preventing the aggregation but not in renaturing denatured proteins. Through incorporating the redox system, we found that $1.2 \mathrm{mM}$ GSSG alone showed a better refolding capability than $1.2 \mathrm{mM} \mathrm{GSH}$ alone. In addition, relative to no activity was measured at $3 \mathrm{Mm} \mathrm{GSH} / 0.3 \mathrm{mM}$ GSSG, the condition with redox reagents at $1.2 \mathrm{mM} \mathrm{GSH} / 1.2 \mathrm{mM}$ GSSG gave the best renaturation performance $\left(0.4 \mathrm{mg} \mathrm{ml}^{-1}\right.$ active lysozyme). Importantly, our data suggest a strong dependency of activity recovery on disulfide bond formation enhanced via the addition of redox reagents. Our data also imply the existence of optimum condition for the collaboration of the oxidized as well as reduced forms in forming correct disulphide bonds and thus restoring the protein activity.

\section{Effect of Constituents on Refolding of Denatured Lysozyme via Size Exclusion Chromatography}

Size exclusion chromatography (SEC) has proven to be a decent technique in refolding denatured proteins due to the fact that the removal of denaturants and the separation of folding intermediates can be conducted simultaneously (Batas et al., 1997; Batas and Chaudhuri, 1996; Jungbauer et al., 2004; Lanckriet and Middelberg, 2004; Liu and Chang, 2003; Wang et al., 2006). In order to dynamically provide a favourable refolding environment for denatured lysozymes, in this work we carried out SEC refolding with the implementation of various GSH/GSSG linear gradient strategies.

\section{Mobile phases are $1.8 \mathrm{mM}$ GSSG and $3.6 \mathrm{mM} \mathrm{GSH}$}

Figure 1 illustrates the chromatographic refolding curves with mobile phase composition linearly changed from $1.8 \mathrm{mM}$ GSSG to $3.6 \mathrm{mM}$ GSH (setting 1). Mobile phase from $3.6 \mathrm{mM}$ GSH to $1.8 \mathrm{mM}$ GSSG (setting 2) was also carried out with different gradient durations, 1, 5, 10 and $20 \mathrm{~min}$, in our SEC refolding experiments. Obviously, the retention time for the refolded protein peak remained unchanged while the environment that denatured proteins passed through was dependent on the gradient duration (see Figure 1). For example, $1 \mathrm{~min}$ of gradient duration allowed lysozyme to completely pass the gradient environment while 20 min permitted protein to experience the first half of the gradient environment. The mass recovery, taken as a refolding performance, was assessed and is shown in Figure 2(a). We found that the mass recovery increased with increasing gradient time as the mobile phase composition changed from $1.8 \mathrm{mM}$ GSSG to $3.6 \mathrm{mM}$ GSH (setting 1). However, the opposite trend was observed when we reversed the composition of mobile phase (setting 2). With an increase in gradient duration, the mass recovery in setting 1 approached to that with the constant final mobile phase composition (isocratic condition that sample injected, i.e., 3.6 mM GSH) [Figure 2(a)]. In addition, our data indicated that, except for the case of 1 min gradient

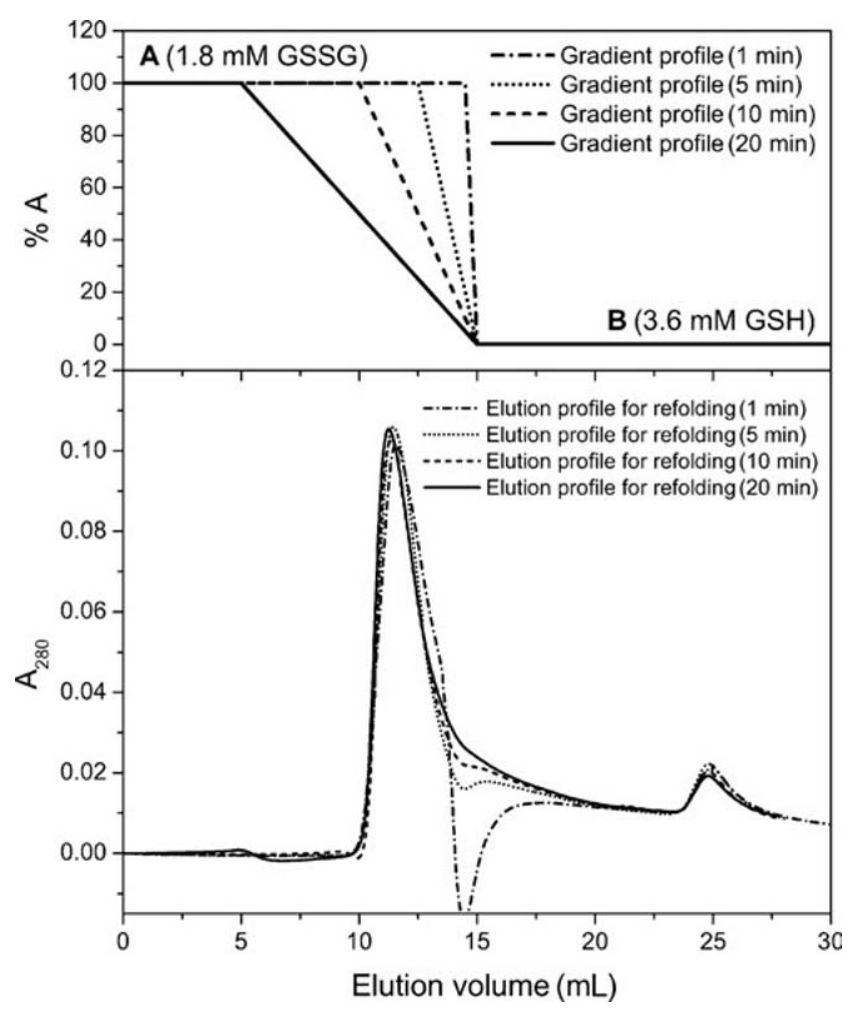

Figure 1. Chromatograms of SEC refolding with mobile phase composition linearly changed from $1.8 \mathrm{mM}$ GSSG to $3.6 \mathrm{mM}$ GSH. The denatured lysozyme at $10 \mathrm{mg} \mathrm{mL}^{-1}$ was introduced into SEC column with linear glutathione gradient. Four different gradient durations, 1, 5, 10 and $20 \mathrm{~min}$, were used in our SEC refolding experiments. *Base line shift due to mobile phase change was adjusted.

time, the setting 1 (injection at $3.6 \mathrm{mM}$ GSH) always allowed more mass recovered than setting 2 (injection at $1.8 \mathrm{mM}$ GSSG), implying the possibility that $3.6 \mathrm{mM}$ GSH placed a better inhibitory action against the formation of incorrect disulphide bonds due to over-oxidation.

Other important renaturation performance index such as the specific activity recovery was also assessed. We show in Figure 2(b) that a tremendous increase in specific activity recovery $(\sim 84 \%)$ was achieved with mobile phase switched from $3.6 \mathrm{mM}$ GSH to $1.8 \mathrm{mM}$ GSSG (setting 2) and gradient time set at $10 \mathrm{~min}$ relative to that at the $3.6 \mathrm{mM}$ GSH isocratically $(\sim 8 \%)$. Our results suggested that the long gradient time led to an elevation of oxidizing power provided by GSSG, and this elevation resulted in the more correct disulphide bonds formed and more activity restored. However, the over-exposure of denatured lysozymes in $1.8 \mathrm{mM}$ GSSG (e.g., gradient time $=20 \mathrm{~min}$ ) could account for a slight descent in the recovery of specific activity $(\sim 80 \%)$. This decrease in activity recovery due to the over-oxidation effect was also strengthened by the fact that lower level of specific activity recovery $(\sim 31 \%)$ was obtained when no GSH was used as the mobile phase.

On the other hand, less specific activity recovery was obtained as the setting 1 was utilized in the operation [Figure 2(b)]. It has been suggested from the literature that the introduction of increasing reducing power (or GSH) could prevent not only the generation of incorrect inter-molecular disulphide bonds (adverse route) but also 

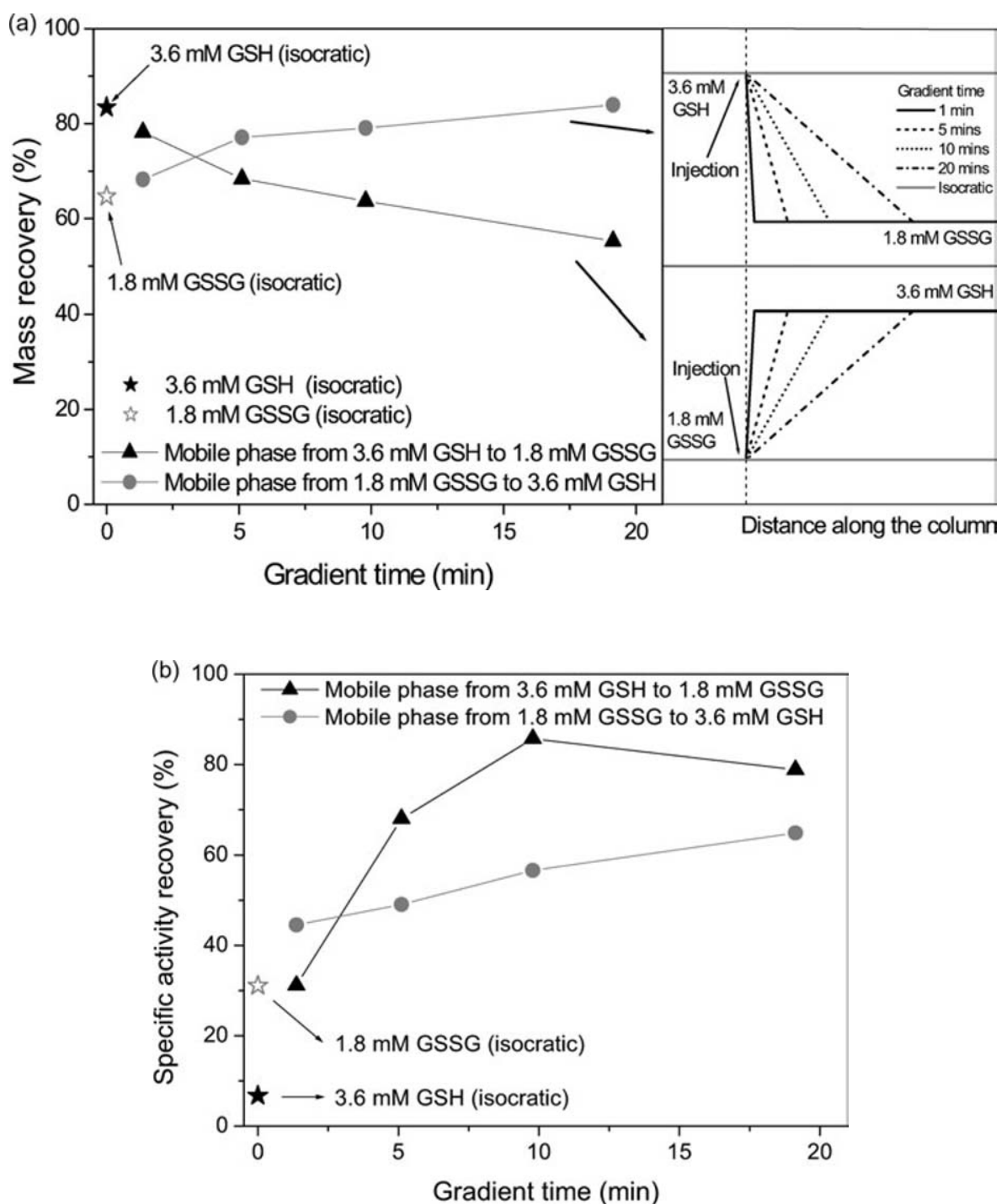

Figure 2. (a) Effect of the gradient time on the enzyme mass recovery via SEC refolding with linear glutathione gradient or isocratic (3.6 mM or $1.8 \mathrm{mM}$ ) operations. (b) Effect of the gradient time on the specific activity recovery via SEC refolding with linear glutathione gradient or isocratic operations. The concentration of denatured lysozyme fed into SEC column was set at $10 \mathrm{mg} \mathrm{mL}^{-1}$.

the formation of correct intra-molecular disulphide bonds (favourable route) (Goto et al., 2000b). Apparently, our results suggest that the favourable pathway dominated in the refolding environment provided by setting 1 .

Mobile phases are $3.0 \mathrm{mM} \mathrm{GSH/0.3} \mathrm{mM}$ GSSG and $1.2 \mathrm{mM}$ GSH/1.2 $\mathrm{mM}$ GSSG

Mobile phase composition linearly changed from $3.0 \mathrm{mM}$ GSH/0.3 mM GSSG to $1.2 \mathrm{mM} \mathrm{GSH} / 1.2 \mathrm{mM}$ GSSG (setting 3) and the reversed operation (setting 4) were investigated, respectively. The recovery of mass was plotted as a function of gradient duration at two different mobile phase settings [see solid circles and triangles in Figure 3(a)]. As shown in Figure 3(a), a slightly higher mass recovery was measured under the setting 4 (injection of protein into $3.0 \mathrm{mM} \mathrm{GSH} / 0.3 \mathrm{mM} \mathrm{GSH}$ ) than that of the setting 3 (injection of protein into $1.2 \mathrm{mM} \mathrm{GSH} / 1.2 \mathrm{mM}$ GSH). No significant difference in the amount of recovered mass was found for various lengths of gradient time in both settings, and even the isocratic operations. Along with our previous results in pure redox reagent settings (Table 1), we postulate that the amount of recovered lysozyme was strongly associated with the aggregates formed during the injection loop. What is important is that the amount of aggregated species appeared to be determined by the constituents of refolding buffer in the mobile phase, especially the conditions of injection, rather than the conditions traveling inside of the column. Therefore, the proper choice of the composition of refolding solution at injection point is the key to preventing the formation of aggregated species. This observation is consistent with our previous findings (Liu and Chang, 2003; Wang et al., 2006).

We also monitored the specific activity recovery as a function of gradient time under the settings 3 and 4 [solid circles and triangles in Figure 3(b)]. We demonstrate in Figure 3(b) that the data from setting 4 exhibited an optimum value at the gradient time of $10 \mathrm{~min}$. Moreover, as contrary to the mass recovery results, the operation at setting 3 showed inferior specific activity recovery to that 

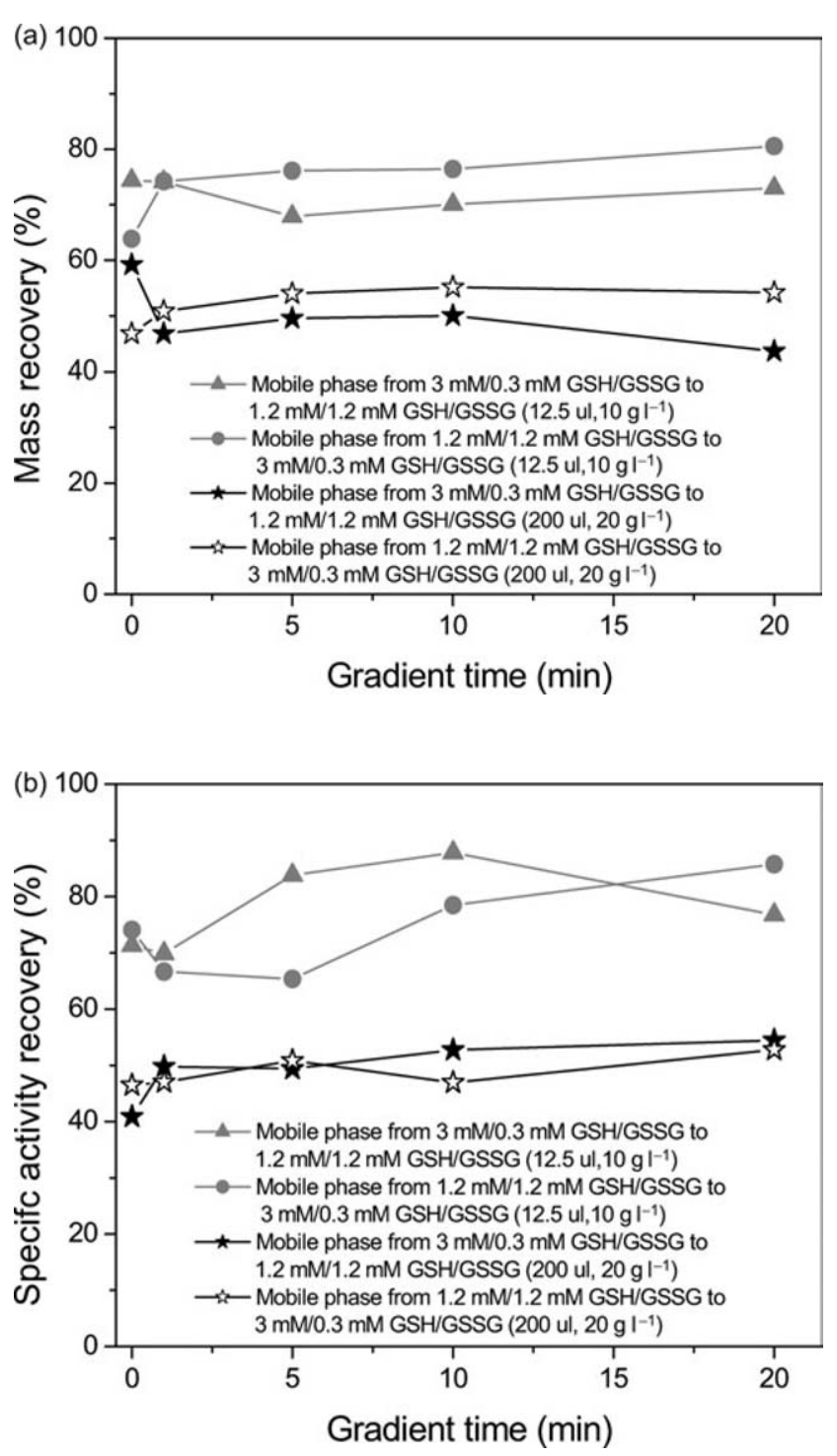

Figure 3. (a) Effect of dilution factor and initial protein concentration on mass recovery via SEC refolding method with different linear glutathione gradient strategies. (b) Effect of dilution factor and initial protein concentration on specific activity recovery via SEC refolding method with different linear glutathione gradient strategies ( $~ 600$-fold: injected volume of $12.5 \mu \mathrm{L}$ and collected volume of $7.5 \mathrm{~mL} ; \sim 43$-fold: injected volume of $200 \mu \mathrm{L}$ and collected volume of $8.5 \mathrm{~mL}$ ).

at setting 4 for the gradient durations at 1,5 and $10 \mathrm{~min}$. The similar behaviour (the occurrence of optimum) was also discovered in SEC refolding under the setting 2.

\section{Mobile phases are 1.2 $\mathrm{mM}$ GSH/1.2 $\mathrm{mM} \mathrm{GSSG}$ and $0 \mathrm{mM}$ GSH/O mM GSSG}

The operations when mobile phase composition linearly changed from $1.2 \mathrm{mM} \mathrm{GSH} / 1.2 \mathrm{mM} \mathrm{GSH}$ to $0 \mathrm{mM}$ GSSG/0 mM GSSG (setting 5) and the reversed operation (setting 6) were also evaluated. The mass recovery of lysozyme is also shown in Figures 4(a). Obviously, no matter which gradient setting was used, almost no change was observed as we increased the duration of gradient. However, the specific activity recovery was found to follow a gradient time-dependent manner [Figure 4(b)]. It has been found only in the setting 6 that a positive correlation existed between the increase in gradient duration and the enhancement of recovered specific activity.

\section{Effect of Dilution Factor and Initial Protein Concentration on SEC Refolding Method with Linear Redox Gradient}

Results from our laboratory indicated that the relative distribution between GSH and GSSG had an influence on refolding performance in direct dilution method. Moreover, this influence became considerable with lower dilution factors. To probe how dilution factor as well as protein concentration impacted the SEC renaturation, we performed the same refolding experiments as settings 3 and 4 by changing sample volume (dilution factor) and protein concentration. The dilution factor in SEC was calculated as the ratio of the eluted volume with protein signal to volume of the injected sample. Besides, for both methods, the duration of refolding process was set at 40 min simply because it was required for SEC to elute all the proteins. Our data indicated that, regardless of the dilution factor (43 or 600 -fold) and injected protein concentration (10 or $20 \mathrm{mg} \mathrm{ml}^{-1}$ ) in use, the profiles of recovery of mass or (specific) enzyme activity as a function of gradient time remained to be unaffected in both settings 3 and 4 [Figure 3(a) and 3(b)].

\section{Comparisons between Direct Dilution and SEC Modes}

We used refolding buffer compositions as a parameter to compare the performance from both direct dilution and SEC strategies and then explore the difference(s) between these two modes of refolding processes. When the initial lysozyme concentration was at $10 \mathrm{mg} \mathrm{ml}^{-1}$ and the dilution factor at 600 , regardless of the refolding methods used, the activity recovery in the cases of combination of reducing and oxidizing reagents employed in the refolding buffer were superior to those in other cases [Figure 5(a)]. Evidently, as opposed to the case with either $3.6 \mathrm{mM}$ GSH or $1.8 \mathrm{mM}$ GSSG, or without any redox reagent, almost no difference in recovered activities was observed between SEC and direct dilution methods in the cases of combination of reducing and oxidizing reagents. However, as shown in Figure 5(b), with the mixed redox reagents used as refolding buffer systems, the discrepancy, from the aspect of activity recovery, between SEC and direct dilution methods became dramatic as the dilution factor was reduced to 43 .

A few points can be made from our results: (1) in the direct dilution method, the likelihood of forming aggregates resulted from lower dilution factor (or higher protein concentration) can be suppressed via an appropriate ratio between redox reagents in the refolding buffer. Our data suggest that $1.2 \mathrm{mM}$ GSH/1.2 mM GSSG (activity recovery $=45 \%$ ) was better than $3.0 \mathrm{mM} \mathrm{GSH} / 0.3 \mathrm{mM}$ GSSG (activity recovery $=15 \%$ ); (2) unlike the direct dilution method, the refolding performance of SEC method was not appreciably reduced in the bad $(3.0 \mathrm{mM} \mathrm{GSH} /$ $0.3 \mathrm{mM}$ GSSG) refolding environment, suggesting the possible role of SEC porous packing in promoting the correct folding of denatured proteins and thus enhancing the 

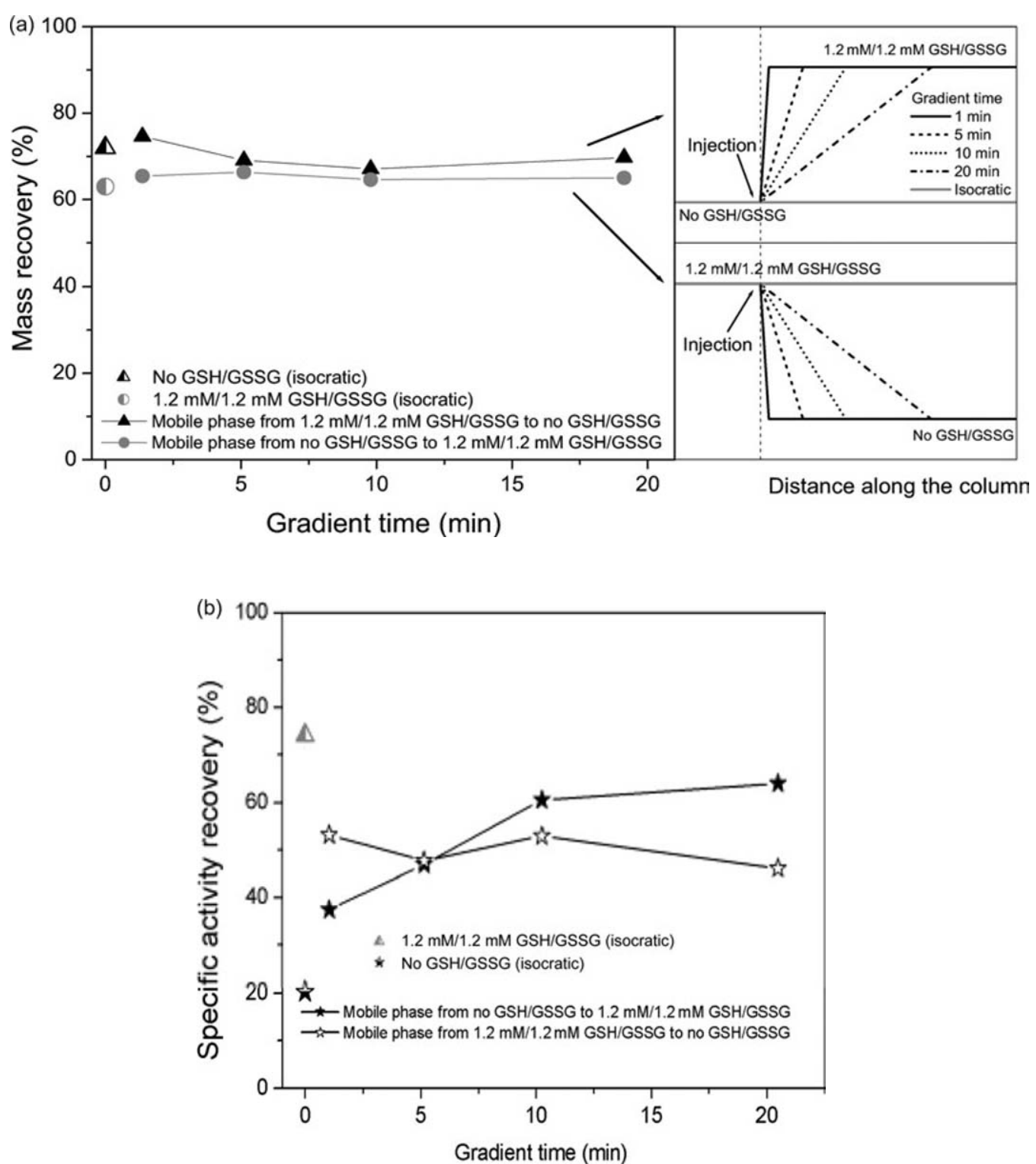

Figure 4. (a) Effect of the gradient time on the enzyme mass recovery via SEC refolding with linear glutathione gradient or isocratic operations. (b) Effect of the gradient time on the specific activity recovery via SEC refolding with linear glutathione gradient or isocratic operations. The concentration of denatured lysozyme fed into SEC column was set at $10 \mathrm{mg} \mathrm{mL}^{-1}$.

refolding performance. (3) SEC was shown to be advantageous over direct dilution method in the absence of GSH/GSSG.

\section{DISCUSSION AND CONCLUSIONS}

The refolding step is of particular importance for restoring the biological activities of industrially produced protein aggregates such as inclusion bodies. Various methods, including the direct dilution and SEC methods (Batas and Chaudhuri, 1996; Chang et al., 2004; Hamaker et al., 1996; Kweon et al., 2004; Lai and Middelberg, 2002; Li and Su, 2002a, b; Li et al., 2002; Liu and Chang, 2003; Wang et al., 2005; Wright and Serpersu, 2004), along with a few strategies (Clark, 2001; Cleland et al., 1992a, b; Dong et al., 2000, 2004; Mannen et al., 2001; Yasuda et al., 1998), have been employed to improve the efficiency/performance of the refolding process; however, refinement and improvement on these methods are still ongoing.

In general, refolding of denatured protein samples can be accomplished either by direct dilution or by column-based (e.g., SEC) scheme. The widely used direct dilution refolding is a simple but buffer-consuming process. Therefore, there are some concerns for large-scale production. On the other hand, in addition to the simultaneous refolding and separation in one unit, further combined with various improved column-based techniques (e.g., annular chromatography), the SEC refolding may be potentially feasible for automation and scalability.

Using the direct dilution method, we first identified the role of urea in the protein refolding process. Our data indicated that low concentrations $(\sim 2 \mathrm{M})$ of urea in the refolding buffer significantly prevented the formation of 

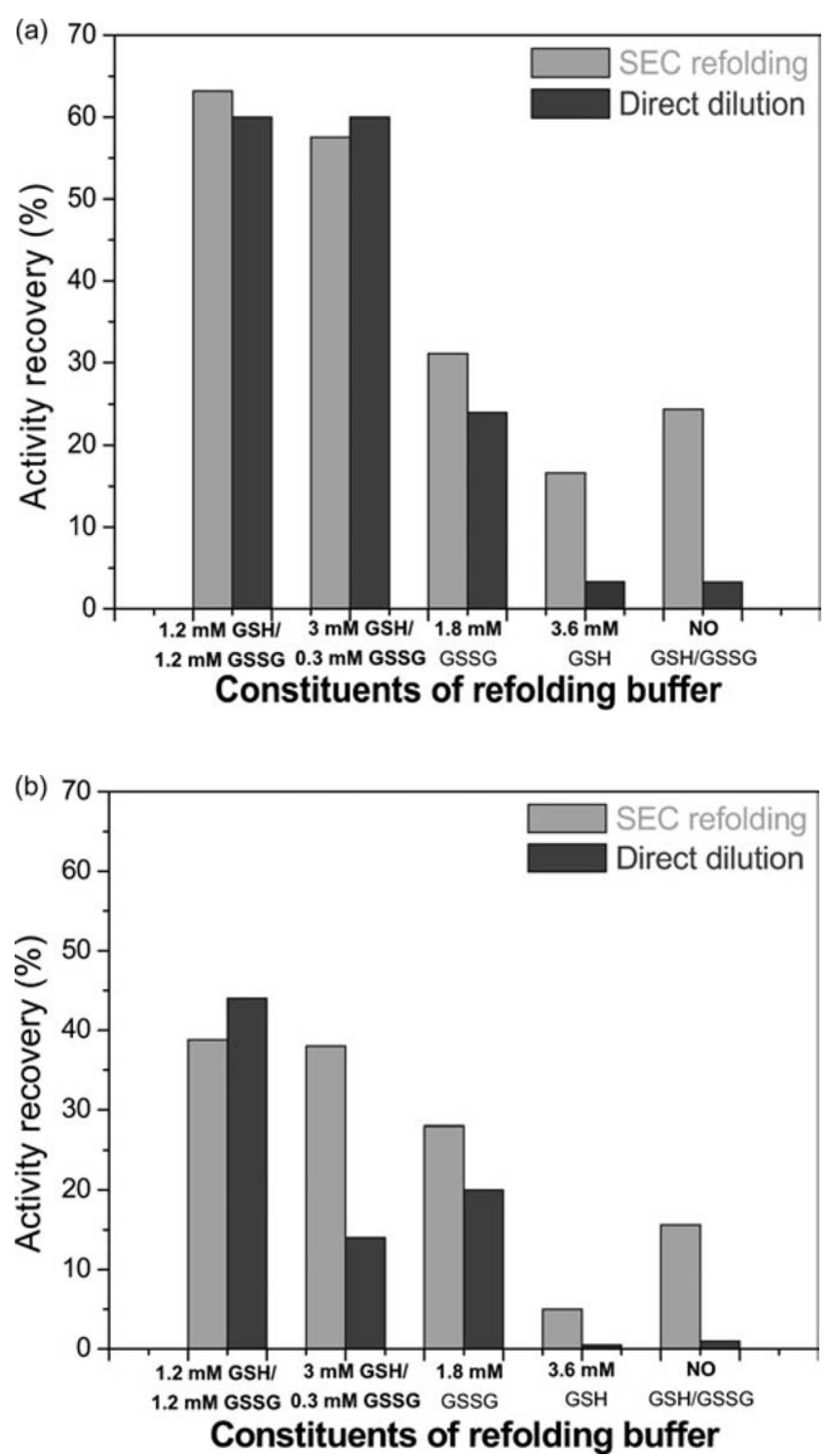

Figure 5. (a) Effect of glutathione redox constituents on specific activity recovery via SEC refolding method with isocratic operation or direct dilution method. A $12.5 \mu \mathrm{L}$ of denatured lysozyme at $10 \mathrm{mg} \mathrm{mL}^{-1}$ was fed into refolding buffer solution. The dilution factor and renaturation duration were set at 600 -fold and $40 \mathrm{~min}$. (b) Effect of glutathione redox constituents on specific activity recovery via SEC refolding method with isocratic operation or direct dilution method. A $200 \mu \mathrm{L}$ of denatured lysozyme at $20 \mathrm{mg} \mathrm{mL}^{-1}$ was fed into refolding buffer solution. The dilution factor and renaturation duration were set at 43 -fold and $40 \mathrm{~min}$.

aggregated species. This conclusion coincides with the evidence reported by others that the presence of a trace amount of urea helps increase the recovery efficiency by attenuating the amount of aggregates that form (Katoh et al., 1999). However, no activity recovery was observed after the addition of urea in the refolding buffer. It has been suggested by our group and others that protein refolding has a deep connection with the formation of 'correct' disulphide bonds through the use of redox reagents (Buczek et al., 2004, 2005; Bulaj, 2005; Bulaj et al., 2004; Fischer et al., 1993; Fischer 1994; Fuller et al., 2005; Zapun and Creighton, 1994). We then characterized the effect of redox reagents on protein refolding performance and clearly demonstrated that the relative distribution between redox counterparts determined the (specific) activity recovery of denatured lysozymes.

A variety of evidence suggests that size exclusion chromatography is a fairly useful technique for protein refolding purposes (Batas and Chaudhuri, 1996; Gu et al., 2001; Jungbauer et al., 2004; Lanckriet and Middelberg, 2004; Liu and Chang, 2003). As a continuation of the statement mentioned above, we were therefore interested in examining how renaturation performance of SEC method changed with composition of redox reagents in refolding buffer. In order to dynamically adjust the refolding environment, the introduction of combination of reduced and oxidized forms of glutathione (GSH and GSSG) and the use of various gradient durations were employed in our SEC refolding experiments. Via varying the ratio of GSH and GSSG, we measured mass recovery and specific activity recovery along with gradient time. We found that, with the GSH/GSSG redox counterparts utilized in mobile phase buffers, the specific activity recovery was elevated upon incorporating the linear gradient strategy in comparison with the corresponding isocratic operations.

To be more specific, several important points can be addressed from our findings. First, the presence of GSH or GSSG, reagents associated with disulphide bridge formation, certainly increased the recovery of specific activity. In addition, the better injection environment of denatured proteins in SEC was confirmed to be a refolding buffer solution containing GSSG not GSH. Ample evidence has implied the close relationship between the formation of native or accurate disulphide bonds, those found within properly folded proteins, and protein stability, function, as well as activity (Bulaj, 2005). It has been proposed that the creation of a single protein disulphide bond is considered as a two consecutive thiol/disulphide exchange step process. The so-called mixed disulphide intermediates, originated from the reaction between thiolate and a low molecular weight disulphide, can be attacked competitively by the intramolecular or intermoleculare thiolates resulting in correctly folded proteins or oligomeric by-products, respectively. The competition between these two routes is thought to be governed by the relative distribution of redox pairs (for example GSH and GSSG). Here, we hypothesize that higher refolding performance from GSSG could be attributed to its function as an oxidative folding enhancer aiding in the improvement of refolding yield. However, possibly due to the fact that the increasing reducing power via GSH could prevent not only the formation of incorrect inter-molecular disulphide bonds but also the generation of correct intra-molecular disulfide bonds, lower refolding efficiency from GSH was observed. Furthermore, a limited efficiency on protein refolding process was observed with either GSH or GSSG reagent alone.

Second, SEC refolding operations with mobile phase composition linearly changed between $3.0 \mathrm{mM} \mathrm{GSH} /$ $0.3 \mathrm{mM}$ GSSG and 1.2 mM GSH/1.2 mM GSSG exhibited superior refolding performances relative to those in other conditions. Also, no abrupt change in specific activity was observed over the range of gradient times when the combination of redox pairs was used. We suspect that the co-existence of reduced and oxidized forms of glutathione offers a suitable and mild environment for a series of thiol/disulphide exchange reactions. Apparently, our current data demonstrate the advantage of using both GSSG and GSH 
possibly owing to their abilities of oxidizing thiolates and reducing incorrect disulphide bonds, respectively.

To further our understanding of the nature of SEC refolding with GSH/GSSG gradient, we examined dilution factor or protein concentration dependence of the refolding performance. Regardless of the ratios of GSH and GSSG in use, the specific activity recovery-gradient time profile was insensitive to changes in dilution factor. Actually, this phenomenon was fairly different from what we observed in the direct dilution refolding.

Lastly, we mechanistically investigated the distinction between the effects of constituents of refolding buffer solution on both SEC refolding and direct dilution refolding operations. In the cases with no or one redox reagent, SEC refolding was shown to be advantageous over direct dilution refolding, indicating the possible function contributed by the SEC packing. In addition, as opposed to the direct dilution method, with porous packing utilized in the SEC column, the contribution of using combination redox system for promote refolding became insignificant. Lanckriet and Middelberg (2004) proposed that, in SEC refolding process, the porous packing provided denatured proteins with nothing but the environment of continuous dilution.

Lanckriet and Middelberg (2004) addressed an issue of DTT carryover effect on the comparisons between direct dilution refolding and SEC refolding. It is the nature of SEC that separation and buffer exchange can be achieved during the process. Due to its different molecular weight, DTT can be separated from other compounds in SEC without extra buffer exchange step. Therefore, no carry-over effect of DTT arises for the case of SEC refolding. As for the direct dilution system, under the dilution conditions, 43- and 600-fold dilutions, used in our study, the concentrations of DTT in use were approximately 0.233 and $0.017 \mathrm{mM}$. According to their work, the effect of DTT carryover from the denatured buffer solution became dramatic as the final DTT concentration after refolding was above $1 \mathrm{mM}$, which is much higher than the ones in our study $(\sim 0.233$ and $\sim 0.017 \mathrm{mM})$. As a result, it is reasonable to assume that the effect of DTT carryover was minor in our direct dilution results.

In summary, while it is unclear from our results as to the reason why the porous packing in refolding enhancement, the outcome from our work did point out that the presence of packing matrices, especially at lower dilution factor, undoubtedly played a key role in promoting the renaturation performance.

\section{REFERENCES}

Batas, B., Jones, H.R. and Chaudhuri, J.B., 1997, Studies of the hydrodynamic volume changes that occur during refolding of lysozyme using size-exclusion chromatography, Journal of Chromatography A, 766: $109-119$.

Batas, D. and Chaudhuri, J.B., 1996, Protein refolding at high concentration using size-exclusion chromatography, Biotechnology and Bioengineering, 50: 16-23.

Buchner, J., Pastan, I. and Brinkmann, U., 1992, A method for increasing the yield of properly folded recombinant fusion proteins: single-chain immunotoxins from renaturation of bacterial inclusion bodies, Anal Biochem, 205: 263-270.

Buczek, O., Olivera, B.M. and Bulaj, G., 2004, Propeptide does not act as an intramolecular chaperone but facilitates protein disulfide isomeraseassisted folding of a conotoxin precursor, Biochemistry, 43: 1093-1101.
Buczek, P., Buczek, O. and Bulaj, G., 2005, Total chemical synthesis and oxidative folding of delta-conotoxin PVIA containing an $\mathrm{N}$-terminal propeptide, Biopolymers, 80: 50-57.

Bulaj, G., 2005, Formation of disulfide bonds in proteins and peptides, Biotechnol Adv, 23: 87-92.

Bulaj, G., Koehn, R.E. and Goldenberg, D.P., 2004, Alteration of the disulfide-coupled folding pathway of BPTI by circular permutation, Protein Science, 13: 1182-1196.

Chang, C.C., Yeh, X.C., Lee, H.T., Lin, P.Y. and Kan, L.S., 2004, Refolding of lysozyme by quasistatic and direct dilution reaction paths: A first-order-like state transition, Physical Review E, 70: 011904

Clark, E.D., 2001, Protein refolding for industrial processes, Current Opinion in Biotechnology, 12: 202-207.

Clark, E.D., Hevehan, D., Szela, S. and Maachupalli-Reddy, J., 1998, Oxidative renaturation of hen egg-white lysozyme. Folding vs aggregation, Biotechnology Progress, 14: 47-54.

Cleland, J.L., Builder, S.E., Swartz, J.R., Winkler, M., Chang, J.Y. and Wang, D.I., 1992a, Polyethylene glycol enhanced protein refolding, Biotechnology (N Y), 10: 1013-1019.

Cleland, J.L., Hedgepeth, C. and Wang, D.I., 1992b, Polyethylene glycol enhanced refolding of bovine carbonic anhydrase B. Reaction stoichiometry and refolding model, J Biol Chem, 267: 13327-13334.

Dong, X.Y., Huang, Y. and Sun, Y., 2004, Refolding kinetics of denaturedreduced lysozyme in the presence of folding aids, $J$ Biotechnol, 114: $135-142$.

Dong, X.Y., Yang, H. and Sun, Y., 2000, Lysozyme refolding with immobilized GroEL column chromatography, Journal of Chromatography A, 878: 197-204.

Fischer, B., Sumner, I. and Goodenough, P., 1993, Isolation, renaturation, and formation of disulfide bonds of eukaryotic proteins expressed in Escherichia-coli as inclusion-bodies, Biotechnology and Bioengineering, 41: 3-13.

Fischer, B.E., 1994, Renaturation of recombinant proteins produced as inclusion-bodies, Biotechnology Advances, 12: 89-101.

Frand, A.R., Cuozzo, J.W. and Kaiser, C.A., 2000, Pathways for protein disulphide bond formation, Trends Cell Biol, 10: 203-210.

Fuller, E., Green, B.R., Catlin, P., Buczek, O., Nielsen, J.S., Olivera, B.M. and Bulaj, G., 2005, Oxidative folding of conotoxins sharing an identical disulfide bridging framework, Febs Journal, 272: 1727-1738.

Goldberg, M.E., Rudolph, R. and Jaenicke, R., 1991, A kinetic-study of the competition between renaturation and aggregation during the refolding of denatured reduced egg-white lysozyme, Biochemistry, 30: 2790-2797.

Goto, M., Fujita, T., Sakono, M. and Furusaki, S., 2000a, Novel protein refolding by reversed micelles, in Endo, I., Nagamune, T., Katoh, S. and Yonemoto, T. (eds). Bioseparation Engineering (Elsevier Science B. V, Amsterdam, The Netherlands.)

Goto, M., Hashimoto, Y., Fujita, T., Ono, T. and Furusaki, S., 2000b, Important parameters affecting efficiency of protein refolding by reversed micelles, Biotechnology Progress, 16: 1079-1085.

Gu, Z.Y., Su, Z.G. and Janson, J.C., 2001, Urea gradient size-exclusion chromatography enhanced the yield of lysozyme refolding, Journal of Chromatography A, 918: 311-318.

Hamaker, K.H., Liu, J.Y., Seely, R.J., Ladisch, C.M. and Ladisch, M.R., 1996, Chromatography for rapid buffer exchange and refolding of secretory leukocyte protease inhibitor, Biotechnology Progress, 12: 184-189.

Hevehan, D.L. and Clark, E.D., 1997, Oxidative renaturation of lysozyme at high concentrations, Biotechnology and Bioengineering, 54: 221230.

Jungbauer, A., Kaar, W. and Schlegl, R., 2004, Folding and refolding of proteins in chromatographic beds, Curr Opin Biotechnol, 15: 487494.

Karuppiah, N. and Sharma, A., 1995, Cyclodextrins as protein folding aids, Biochem Biophys Res Commun, 211: 60-66.

Katoh, S., Sezai, Y., Yamaguchi, T., Katoh, Y., Yagi, H. and Nohara, D., 1999, Refolding of enzymes in a fed-batch operation, Process Biochemistry, 35: 297-300.

Kim, S., Baum, J. and Anderson, S., 1997, Production of correctly folded recombinant C-13, N-15-enriched guinea pig Val90-alpha-lactalbumin, Protein Engineering, 10: 455-462.

Kweon, D.H., Lee, D.H., Han, N.S. and Seo, J.H., 2004, Solid-phase refolding of cyclodextrin glycosyltransferase adsorbed on cationexchange resin, Biotechnol Prog, 20: 277-283.

Lai, W.B. and Middelberg, A.P.J., 2002, The production of human papillomavirus type $16 \mathrm{~L} 1$ vaccine product from Escherichia coli inclusion bodies, Bioprocess and Biosystems Engineering, 25: 121-128. 
Lanckriet, H. and Middelberg, A.P., 2004, Continuous chromatographic protein refolding, J Chromatogr A, 1022: 103-113.

Li, M. and Su, Z., 2002a, Refolding human lysozyme produced as an inclusion body by urea concentration and $\mathrm{pH}$ gradient ion exchange chromatography, Chromatographia, 56: 33-38.

Li, M. and Su, Z.G., 2002b, Refolding of superoxide dismutase by ionexchange chromatography, Biotechnology Letters, 24: 919-923.

Li, M., Zhang, G.F. and Su, Z.G., 2002, Dual gradient ion-exchange chromatography improved refolding yield of lysozyme, Journal of Chromatography A, 959: 113-120.

Liu, H.S. and Chang, C.K., 2003, Chaperon solvent plug to enhance protein refolding in size exclusion chromatography, Enzyme and Microbial Technology, 33: 424-429.

Maeda, Y., Ueda, T. and Imoto, T., 1996, Effective renaturation of denatured and reduced immunoglobulin $\mathrm{G}$ in vitro without assistance of chaperone, Protein Engineering, 9: 95-100.

Mannen, T., Yamaguchi, S., Honda, J., Sugimoto, S. and Nagamune, T., 2001, Expanded-bed protein refolding using a solid-phase artificial chaperone, Journal of Bioscience and Bioengineering, 91: 403-408.

Negro, A., Onisto, M., Grassato, L., Caenazzo, C. and Garbisa, S., 1997, Recombinant human TIMP-3 from Escherichia coli: Synthesis, refolding, physico-chemical and functional insights, Protein Engineering, 10: $593-599$.

Wang, S.S.-S., Chang, C.K. and Liu, H.S., 2006, Step Change of Mobile Phase Flow Rates to Enhance Protein Folding in Size Exclusion Chromatography, Biochemical Engineering Journal, (in press):
West, S.M., Chaudhuri, J.B. and Howell, J.A., 1998, Improved protein refolding using hollow-fibre membrane dialysis, Biotechnology and Bioengineering, 57: 590-599.

Wetlaufer, B., D. and Y., X., 1995, Control of aggregation in protein refolding nucleotidyltransferase (2")-Ia from inclusion bodies as active, monomeric enzyme, Protein Expression and Purification, 35: 373-380.

Wright, E. and Serpersu, E.H., 2004, Isolation of aminoglycoside nucleotidyltransferare( $\left.2^{\prime \prime}\right)$-Ia from inclusion bodies as active, monomeric enzyme.

Yasuda, M., Murakami, Y., Sowa, A., Ogino, H. and Ishikawa, H., 1998, Effect of additives on refolding of a denatured protein, Biotechnology Progress, 14: 601-606.

Zapun, A. and Creighton, T.E., 1994, Effects of Dsba on the disulfide folding of bovine pancreatic trypsin-inhibitor and alpha-lactalbumin, Biochemistry, 33: 5202-5211.

Zardeneta, G. and Horowitz, P.M., 1994, Protein refolding at high concentrations using detergent/phospholipid mixtures, Anal Biochem, 218: 392-398.

\section{ACKNOWLEDGEMENTS}

This work was supported by grants from the National Science Council, Taiwan.

The manuscript was received 7 July 2005 and accepted for publication after revision 20 February 2006. 